Conclusion: We assessed whether the association between BMI and OA was mediated by hypertension and atherosclerosis. Our results imply that either such mediation is absent or trivial, or that the atherosclerosis measures were too weak.

Disclosure of Interests: None declared

DOI: 10.1136/annrheumdis-2020-eular.3080

\section{FRI0414 FACTORS ASSOCIATED WITH THE IMPACT OF GONARTHROSIS ON MUSLIM PRAYER}

M. Hfaidh ${ }^{1}$, K. Maatallah ${ }^{1}$, H. Ferjani ${ }^{1}$, W. Triki ${ }^{1}$, D. Kaffel ${ }^{1}$, W. Hamdi ${ }^{1}$. ${ }^{1}$ Mohamed Kassab Institute of Orthopedics, Rheumatology Department, Manouba, Tunisia

Background: Gonarthrosis is a frequent and chronic pathology, which can cause painful functional impotence and limit the performance of activities of daily living[1].

Objectives: This study aimed to assess the patient's perception of the physical and psychological repercussions of his illness on the practice of prayer and to determine the factors associated with this repercussion.

Methods: It was a cross-sectional prospective study conducted in the rheumatology department in 56 patients with gonarthrosis who regularly practiced prayer before the onset of the disease. The socio-demographic data, the clinical characteristics of gonarthrosis were studied and a pre-established questionnaire was offered to patients to assess the physical and psychological impact of gonarthrosis on their prayer practice.

Results: Fifty-six patients were included, $83.3 \%$ of whom were female. The average age was 56.1 years [38-78 years]. The disease has progressed for an average of 6.14 years [ $1-13$ years]. Gonarthrosis was bilateral in $80.4 \%$ of cases. The average body mass index (BMI) was $30.29 \mathrm{~kg} / \mathrm{m}^{2} \pm 3.061$ with extremes ranging from 24 to $36 \mathrm{~kg} / \mathrm{m}^{2}$. Quadriceps $(\mathrm{Q})$ retraction was noted in $64.28 \%$ of cases. Gonarthrisis was classified as stages I, II and III according to the classification of Kellegren and Lawrence in $14.3 \%, 57.1 \%$ and $28.6 \%$ of patients respectively.

In $71.4 \%$ of cases (40 patients), the practice of prayer after the onset of gonarthrosis was considered more difficult with a degree of difficulty of 4.23/10 \pm 2 . Initial standing was considered possible by all patients. Inclination was possible in $89.2 \%$ of patients, whereas it was replaced by sitting on a chair by the rest. Prostration and final sitting station were considered impossible by $64.3 \%$ of patients and were therefore performed on chairs (36 patients). The limiting factor cited by patients was pain in $100 \%$ of cases. A psychological impact was reported in $53.6 \%$ of cases. It was explained by the feeling of guilt in 22 cases, the relatives' comments in 8 cases and the suffering related to disability in 7 cases.

Prayer position was associated with $Q$ retraction $(p=0.001)$ and knee pain seniority $(6.81 \pm 3.608 v s 4.95 \pm 3.017, p=0.05)$. The degree of difficulty was associated with the BMI $(p=0.013)$, knee pain seniority $(p<0.001)$ and $Q$ retraction $(5.74 \pm 1.851$ vs $3.46 \pm 1.789, p<0.001)$.

Conclusion: Prayer is an activity that is part of the daily lives of many Muslim patients, its evaluation should be considered as one of the elements of the quality of life and functional impact of gonarthrosis.

References:

[1] Leclerc L, Rossignol M. Retentissement fonctionnel de l'arthrose: résultats d'une enquête nationale effectuée auprès de 10000 patients consultant pour arthrose. Rev Rhum Ed Fr. 2005;5:404-10.

Disclosure of Interests: None declared

DOI: 10.1136/annrheumdis-2020-eular.6100

\section{BODY MASS INDEX AND STATIC FOOT DISORDERS IN GONARTHROSIC PATIENTS}

K. Maatallah ${ }^{1}$, M. Hfaidh ${ }^{1}$, H. Ferjani ${ }^{1}$, W. Triki ${ }^{1}$, D. Kaffel ${ }^{1}$, W. Hamdi ${ }^{1}$.

${ }^{1}$ Mohamed Kassab Institute of Orthopedics, Manouba, Tunisia

Background: Several studies have shown that there is a link between body mass index (BMI) and painful foot imputed to a biomechanical change in foot structure [1].

Objectives: Our objective was to study the association between BMI and static foot disorders in gonarthrosic subjects.

Methods: It was a prospective descriptive study conducted in the rheumatology department of the Mohamed Kassab Institute of Orthopedics with 60 patients with Gonarthrosis. The socio-demographic data of the patients were studied. BMI was calculated for all patients. Static foot disorders have been studied.

Results: Sixty patients were included, $83.3 \%$ of whom were female. The average age was 55.2 years [38-78 years]. The disease has been evolving for an average of 6 years [ $1-13$ years]. The lesion was bilateral in $80 \%$ of cases, the average body mass index was $30.4 \mathrm{~kg} / \mathrm{m} 2$ [24-36]. Knee arthritis was classified as stage I, II and III according to the Kellgren and Lawrence classification in 18.5\%, 55.6\% and $25.9 \%$ of patients respectively. The foot examination involved 108 gonar throsic limbs. Examination of the integuments showed hyperkeratosis in $94.4 \%$ of the cases $(79.6 \%$ calluses and $83.3 \%$ callosities). Forefoot deformities were Hallux valgus (HV) in $52.8 \%$ of cases and overlapping toes in $18.5 \%$ of cases. Pronation deformity using the Foot Posture Index (FPI) was found in $51.9 \%$ of cases. Abnormal lowering of navicular bone was noted in $51.9 \%$. The podoscopic impression revealed flat feet in $73.2 \%$ of the cases.

A statistically significant association was found between BMI and the presence of calluses $(31.21 \pm 2.897$ vs26.83 $\pm 1.425, p<0.001)$, with HV $(31.37$ \pm 3.086 vs29.49 $\pm 2.969, p=0.002)$, at the overlap of the toes $(33.2 \pm$ $1.361 v s 29.86 \pm 1.130, p<0.001)$, with the lowering of the navicular bone $(31.17 \pm 2.885 v s 29.68 \pm 3.304, p=0.015)$, FPI $(p=0.003)$ and flat podoscopic impression $(p<0.001)$.

Conclusion: BMI is strongly associated with static feet disorders in gonarthrosic patients by aggravating the postural changes in the foot caused by knee osteoarthritis [2]. Obesity is associated mainly with the existence of flat feet, pronation of the foot, toes deformities and hyperkeratosis.

References:

[1] Steele JR, Mickle KJ, Munro B. Fat flat frail feet: how does obesity affect the older foot. XXII Congress of the International Society of Biomechanics; 2009

[2] Norton AA, Callaghan JJ, Amendola A, Phisitkul P, Wongsak S, Liu SS, et al. Correlation of knee and hindfoot deformities in advanced knee OA: compensatory hindfoot alignment and where it occurs. Clin Orthop Relat Res. 2015;473(1):166-74

Disclosure of Interests: None declared

DOI: 10.1136/annrheumdis-2020-eular.6337

\begin{tabular}{|l|l}
\hline FRI0416 & COMBINATION OF SERUM ADIPOKINES/RELATED \\
INFLAMMATORY FACTORS AND RATIOS AS \\
PREDICTORS OF INFRAPATELLAR FAT PAD VOLUME \\
IN KNEE OSTEOARTHRITIS PATIENTS: USAGE OF A \\
COMPREHENSIVE MACHINE LEARNING APPROACH
\end{tabular}

H. Bonakdari ${ }^{1,2}$, G. Tardif ${ }^{1}$, F. Abram ${ }^{3}$, J. P. Pelletier ${ }^{1}$, J. Martel-Pelletier ${ }^{1}$. ${ }^{1}$ University of Montreal Hospital Research Centre (CRCHUM), Osteoarthritis Research Unit, Montreal, Canada; ${ }^{2}$ Laval University, Department of Soil and Agri-Food Engineering, Quebec, Canada; ${ }^{3}$ ArthroLab Inc., Medical Imaging Research and Development, Montreal, Canada

Background: One of the hurdles in osteoarthritis (OA) drug discovery and the improvement of therapeutic approaches is the early identification of patients who will progress. It is therefore crucial to find efficient and reliable means of screening OA progressors. Although the main risk factors, age, gender and body mass index (BMI), are important, they alone are poor predictors. However, serum factors could be potential biomarkers for early prediction of knee OA progression.

Objectives: In a first step toward finding early reliable predictors of OA progressors, this study aimed to determine, in OA individuals, the optimum combination of serum levels of adipokines/related inflammatory factors, their ratios, and the three main OA risk factors for predicting knee OA infrapatellar fat pad (IPFP) volume, as this tissue has been associated with knee OA onset and progression.

Methods: Serum and magnetic resonance images (MRI) were from the Osteoarthritis Initiative at baseline. Variables (48) comprised the 3 main OA risk factors (age, gender, BMI), 6 adipokines, 3 inflammatory factors, and their 36 ratios. IPFP volume was assessed on MRI with a neural network methodology. The best variables and models were identified in Total cohort $(n=678)$, High-BMI $(n=341)$ and Low-BMI ( $\mathrm{n}=337$ ), using an artificial intelligence selection approach: the adaptive neuro-fuzzy inference system embedded with fuzzy c-means clustering (ANFIS-FCM). Performance was validated using uncertainty analyses and statistical indices. Reproducibility was done using 80 OA patients from a clinical trial (female, $n=57$; male, $n=23$ ).

Results: For the three groups, $8.44 \mathrm{E}+14$ sub-variables were investigated and 48 models were selected. The best model for each group included five variables: the three risk factors and adipsin/C-reactive protein combined for Total cohort adipsin/chemerin; High-BMl, chemerin/adiponectin high molecular weight; and Low-BMI, interleukin-8. Data also revealed that the main form of the ratio used for the model was justified, as the use of the inverse form slightly decreased the performance of the model in both training and testing stages. Further investigation indicated that gender improved (13-16\%) the prediction results compared to the BMI-based models. For each gender, we then generated a pseudocode (an evolutionary computation equation) with the 5 variables for predicting IPFP volume. Reproducibility experiments were excellent (correlation coefficient: female 0.83 , male 0.95) 